\title{
QUASI-ELASTIC NEUTRON SCATTERING FROM LIQUID GALLIUM OVER THE TEMPERATURE RANGE 163 TO $333 \mathrm{~K}$
}

\author{
L. BOSIO \\ C.N.R.S., 4, place Jussieu, T 22, 75230 Paris Cedex 05, France \\ E. SCHEDLER \\ Institut Laue-Langevin, 38042 Grenoble Cedex, France \\ and \\ C. G. WINDSOR \\ A.E.R.E., Harwell, OX11ORA, U.K. \\ (Reçu le 9 janvier 1976, accepté le 16 février 1976)
}

\begin{abstract}
Résumé. - A l'aide d'un spectromètre à trois axes, nous avons étudié la diffusion quasi élastique de gouttelettes de gallium liquide jusqu'à des températures proches de la température minimale $T_{\mathrm{L}}=150 \mathrm{~K}$ à laquelle le liquide peut être maintenu en surfusion. Les mesures ont été essentiellement effectuées au voisinage des valeurs du vecteur d'onde $Q_{0}=2,50 \AA^{-1}$ et $Q_{00}=3,12 \AA^{-1}$ où le facteur de structure $S(Q)$ présente respectivement un maximum principal et un épaulement. A $Q_{0}$, la largeur à mi-hauteur du pic quasi élastique, bien représenté par une Lorentzienne, décroît linéairement en fonction de la température avec une pente égale à $0,0016 \pm 0,0002 \mathrm{THz} \cdot \mathrm{K}^{-1}$ et tend vers zéro à la température $T=157 \pm 10 \mathrm{~K}$. Cette température peut être considérée comme étant la limite en dessous de laquelle le liquide devient instable.
\end{abstract}

Abstract. - Using a triple axis spectrometer we have measured the quasi-elastic scattering from liquid gallium droplets down to near the lowest temperature $T_{\mathrm{L}}=150 \mathrm{~K}$ at which the liquid remains in the supercooled state. Special reference was given to values of the wave-vector $Q_{0}=2.50 \AA^{-1}$ and $Q_{00}=3.12 \AA^{-1}$ corresponding respectively to the mean peak and the shoulder in the structure factor $S(Q)$. At $Q_{0}$ the full width at half height of the Lorentzian profile fitting the quasi-elastic peak is found to vary linearly with temperature below $333 \mathrm{~K}$, with a rate equal to $0.0016 \pm 0.0002 \mathrm{THz}$. $\mathrm{K}^{-1}$ approaching a zero value at $T=157 \pm 10 \mathrm{~K}$ which we associate with the metastability limit of the liquid state.

1. Introduction. - We have studied the neutron inelastic scattering from supercooled gallium to see if there exists a stability limit in the liquid phase [1]. Although gallium is not a simple liquid [2], it was chosen because it may be supercooled over an exceptional temperature range down to close to half its normal melting point $T_{\mathrm{m}}=303 \mathrm{~K}$ [3]. Our neutron measurements were performed at temperatures down to $163 \mathrm{~K}$.

Theories of liquid state have related the stability of the liquid to the dynamics of its collective modes [4 to 10]. For example Schneider et al. have associated the approach of the stability limit to the softening of purely imaginary normal mode frequency $\Omega(Q)$. If for any value of the wave-vector $Q, \Omega(Q)$ approached zero, there would be an instability with respect to density fluctuations of this wave-vector and the liquid would become unstable. However in their model, Schneider et al. related this value of $Q$ to the value $Q_{0}$ corresponding to the maximum in the liquid structure factor $S(Q)$ and showed that $S\left(Q_{0}\right)$ should diverge as the stability limit is approached. There have been several recent experiments measuring $S(Q)$ by X-ray scattering in supercooled liquid gallium $[11,12]$. In particular the experiments of Bizid et al. have been performed at temperatures down to near the lowest temperature at which gallium has ever been obtained in the liquid phase, and show that there is no tendency to any divergence in $S(Q)$. The $\mathrm{X}$-ray results also show that when freezing does occur from these low temperatures, it is always to the crystalline $\beta$-phase. Amorphous gallium has been prepared by vapour 
deposition on helium cooled substrates $[13,14]$ but is observed to be unstable on heating beyond $15 \mathrm{~K}$, transforming again to the crystalline $\beta$-phase; the $S(Q)$ from the amorphous gallium appears similar in form to that in the supercooled liquid.

The time dependance of the density fluctuations may be studied directly by inelastic neutron scattering. The cross-section observed with a momentum transfer $\hbar Q$ and energy transfer $\hbar \omega$ gives the scattering function $S(Q, \omega)$ related to the Fourier transform over space and time of pair correlation function :

$$
\begin{array}{r}
S(Q, \omega)=\frac{1}{2 \pi} \int \mathrm{d} \mathbf{r} \int \exp [i(\mathbf{Q} \cdot \mathbf{r}-\omega t)] \times \\
\times[G(\mathbf{r}, t)-\rho)] \mathrm{d} t
\end{array}
$$

Van Hove's correlation function $G(\mathbf{r}, t)$ has the classical interpretation of the probability that, given an atom initially at the origin, there will be an atom at distance $r$ observed at a time $t$ later. It divides into a distinct term and a self term when the atom observed is different from or the same as that initially observed at the origin. They can be measured experimentally in some exceptional cases when appropriate isotopes are available form the coherent and incoherent scattering cross-section.

The general form of the scattering function $S(Q, \omega)$ from a liquid takes the form of a Lorentzian frequency distribution with widths increasing as $Q^{2}$ in the simple diffusion approximation [15]. However, it is well known that these widths are modulated by the form of the structure factor which measures the total scattering :

$$
S(Q)=\int S(Q, \omega) \mathrm{d} \omega
$$

according to the de Gennes exact relationship between the second moment of $S(Q, \omega)$ and $S(Q)$ :

$$
\left\langle\omega^{2}\right\rangle=\frac{Q^{2} k T}{M S(Q)} .
$$

Thus certainly the divergent $S(Q)$ postulated by Schneider et al. would lead to a vanishing second moment of energy distribution $S(Q, \omega)$.

Several inelastic neutron scattering experiments measuring the widths of $S(Q, \omega)$ as function of $Q$ and temperature have already been performed from liquid gallium $[16,17,18]$. These studies were generally in agreement with one another in yielding half widths, measured near the peak in the structure factor, which decreased much faster than the $T^{1 / 2}$ predicted by eq. (3). However the temperature ranges of these studies were too restricted to enable conclusions to be drawn on any metastability limit. The present inelastic studies have been performed in an extended range of supercooling by using droplet samples.
2. Experimental details. - Sample and cell : It has been known for many years that the use of very small samples lowers the temperature to which gallium may be supercooled. Particle sizes of order $5 \mu \mathrm{m}$ are obtained by using an ultrasonic generator to disperse liquid gallium within alcohol. In our case $99.9999 \%$ pure gallium was dispersed in ethyl alcohol and then washed by deuterated methyl alcohol. After leaving for some hours to settle, much of the alcohol could be decanted off. Some of the alcohol was dried by evaporation from the cell so that the influence of deuterated methyl alcohol was negligible. This is illustrated by figure 1 which shows uncorrected diffraction results $S(Q, \omega=0)$ obtained at $273 \mathrm{~K}$ with bulk and emulsified gallium samples in identical cells. At least for $Q>2.2 \AA^{-1}$ the curves overlap and hence neutron scattering from emulsified liquid gallium may be considered identical to the bulk liquid.

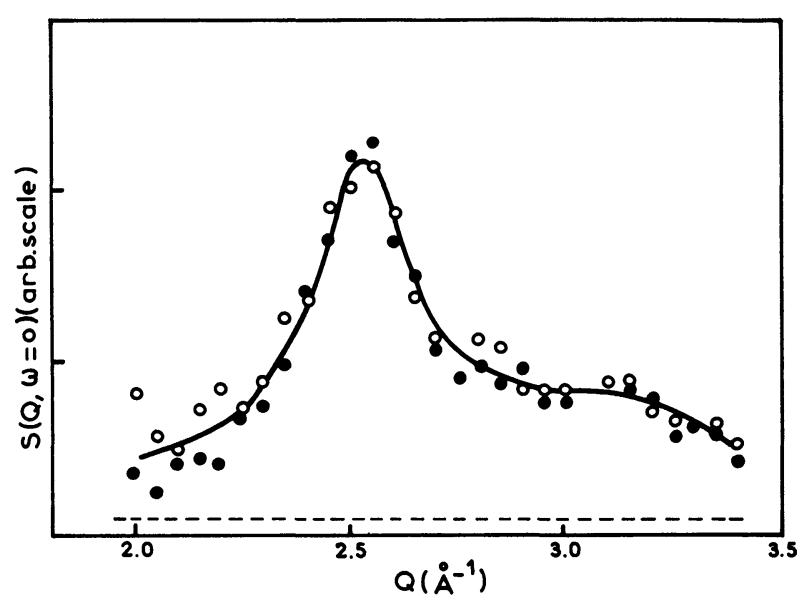

FIG. 1. - Elastic scattering $S(Q, \omega=0)$ from bulk (closed circles) and dispersed liquid gallium (open circles) at $273 \mathrm{~K}$.

Gallium was contained in a square plate cell (40 $\mathrm{mm} \times 40 \mathrm{~mm} \times 3 \mathrm{~mm}$ ) constructed from vanadium sheet $0.125 \mathrm{~mm}$ thick on a stainless steel framework. The cell was put in a cryostat whose temperature could be regulated to within $0.2 \mathrm{~K}$ as measured by a gallium arsenide probe fastened to the specimen. The construction of the cryostat allowed replacement of the cell without breaking the cryostat vacuum; this proved valuable as it was found necessary to replace the sample many times when undesirable freezing occurred prematurely. A sample was successfully cooled to $213 \mathrm{~K}$ without formation of any solid, and particularly detailed measurements were made on this sample. A further sample was cooled to $183 \mathrm{~K}$ and $163 \mathrm{~K}$ with only a small fraction of droplets crystallised in the $\beta$-phase; inelastic scattering measurements at $Q_{0}$ were possible because there is no Bragg's peak at $Q=2.50 \AA^{-1}$. This sample was finally warmed again to $213 \mathrm{~K}$ so that by comparison with the previous measurements the amount of liquid present at 183 and $163 \mathrm{~K}$ could be reliably estimated.

The spectrometer : Our experiments were performed 
on the triple axis spectrometer IN3 at the LaueLangevin Institute at Grenoble [19]. The ability of this spectrometer to allow large angles of scattering at the monochromator and analyser crystals gave good energy resolution without the use of collimators, and yet allowed constant $Q$ scans to be performed with adequate $Q$ resolution. Neutrons are carried to the spectrometer by a curved nickel guide tube which transmits $1.9 \AA$ neutrons with a divergence of the order of $0.3^{\circ}$. Monochromatic neutrons of wavevector $k_{0}=3.30 \AA^{-1} \quad(\lambda=1.904 \AA)$ were selected by the (220) plane of a copper single crystal. The beam area was defined by apertures $40 \mathrm{~mm}$ high by $20 \mathrm{~mm}$ wide. The flat sample was located $1.80 \mathrm{~m}$ from the monochromator and made a constant angle $\Psi=38^{\circ}$ with the incident beam direction. No collimators were used but a flat plate sample at this angle gives a focusing effect for quasi-elastic scattering. This effect is illustrated in figure 2. The spread in the incident

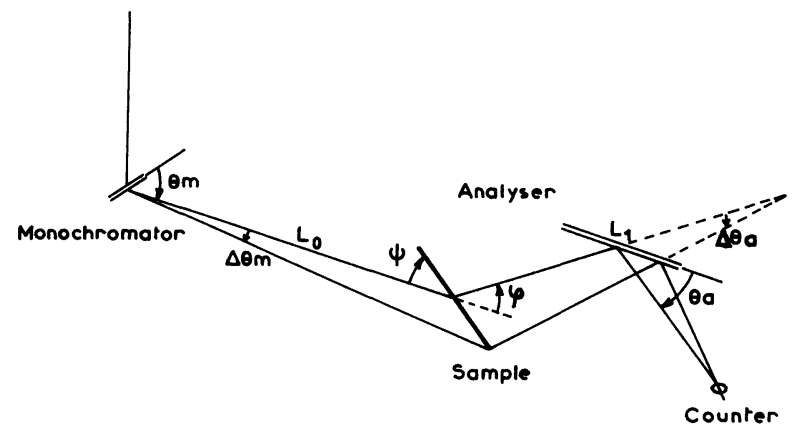

FIG. 2. - The possibility of focusing energy transfers from a finite sized sample recorded by a single counter.

wave-vector magnitude caused by the divergence of the neutron beam and the sample size is given by $\left|\Delta k_{0}\right|=k_{0} \cot \theta_{\mathrm{m}} \Delta \theta_{\mathrm{m}}$. However this will cause no spread in the energy transfer if it is matched against an identical spread in the scattered wave-vector $\left|\Delta k_{1}\right|=k_{1} \cot \theta_{\mathrm{a}} \Delta \theta_{\mathrm{a}}$. This can be achieved with the spectrometer in the $W$ configuration and a scattering angle $\varphi$ if the sample angle $\Psi$ is given by

$$
\cot \Psi=\frac{k_{0}}{k_{1}} \frac{\cot \theta_{\mathrm{m}}}{\cot \theta_{\mathrm{a}}} \cdot \frac{L_{1}}{L_{0}} \cdot \frac{1}{\sin \varphi}-\cot \varphi .
$$

At the two lowest temperatures (183 and $163 \mathrm{~K}$ ) an aperture $12 \mathrm{~mm}$ wide was placed in the scattered beam to improve the resolution. The analyser crystal was $1.0 \mathrm{~m}$ from the sample and employed the (333) plane of germanium in nearly back reflection. The detector system was an array of $24{ }^{3} \mathrm{He}$ filled proportional counters of $10 \mathrm{~mm}$ diameter, $100 \mathrm{~mm}$ high and spaced $15 \mathrm{~mm}$ apart. When located far from the large analyser crystal the array operates like a MARX spectrometer. In this mode, when the central counter of the array is arranged to scan against $Q$ at the elastic energy, the other counters provide a quasi-elastic scan at values of wave-vector slightly different from $Q$. Also when the central counter is performing a quasi-elastic energy scan at constant $Q$, the other counters perform very nearly constant $Q$ scans at slightly displaced energies. The principle of the method is illustrated in figure $3 a$. The finite length of the counter array $2 x$ determines a range of scattered neutron analyser angles $\Delta \theta_{\text {a }}$ giving in turn a range of measured energy transfers. This in turn leads to a change in scattering angles $\Delta \varphi$ so that there is a range in reciprocal space covered as illustrated in the inset of figure $3 a$. This is a line in reciprocal space whose orientation depends on the relative sign of the analyser and sample angles. During a constant $Q$ frequency scan, the centre of the region in reciprocal space covered naturally remains fixed but there will be a change in its orientation. In the configuration used during our experiment the centre 11 counters were employed. They have an effective spacing of $7.5 \mathrm{~mm}$ and an effective sample counter distance $L_{1}$ of $2.16 \mathrm{~m}$. The ranges in $|Q|$ and $\omega$ covered by these counters during a quasi-elastic frequency scan from -0.5 to $0.5 \mathrm{THz}$ are shown in figure $3 b$. It will be seen than while the centre counter, number 6 , has a precisely defined value of $|Q|$, the other counters have slightly different and changing

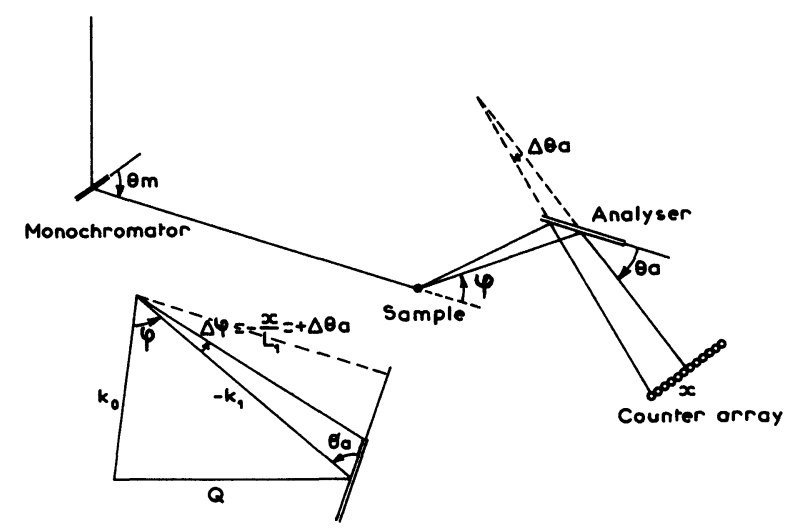

a)

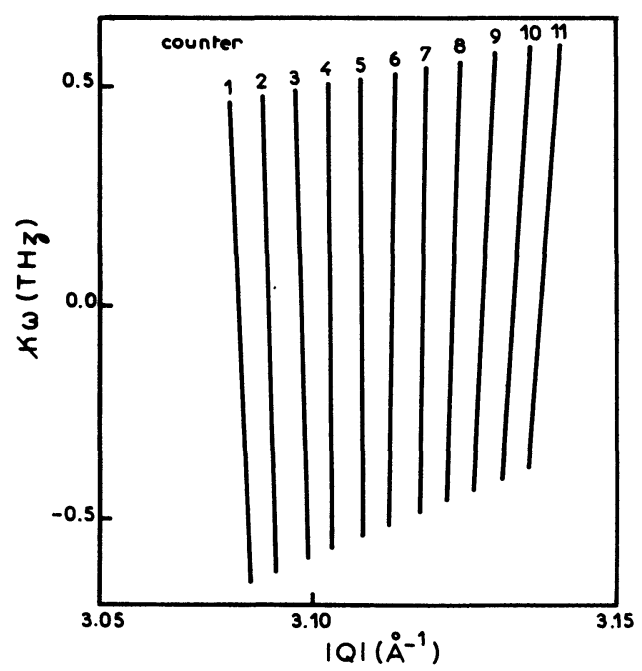

b)

Fig. 3a. - The principle of the multicounter. The range of accepted analyser angles $\Delta \theta_{\mathrm{a}}$ determines the range of reciprocal space covered, shown on the inset. $3 b$. - Range in $Q$ and $\omega$ covered by the counters during a quasi-elastic scan from -0.5 to $0.5 \mathrm{THz}$. 
values of $|Q|$ and are displaced in energy by an amount depending on the position in the scan. The data from all the counters are combined together using a computer code which first calculated the exact values of $|Q|$ and $\omega$ for each counter $i$ and for each point in the scan $j$. A histogram was then compiled centred on the frequencies $\omega_{j}^{0}$ specified in the original constant $Q$ scan. The neutron counts $C_{i j}$ for each counter $i$ were distributed over the histogram boxes, the counts appropriate to the precise frequency $\omega_{i j}$ being linearly distributed between the histogram boxes covering this frequency. The respective monitor counts were similarly distributed so that the points on the histogram could be subsequently normalised. By combining data from eleven central counters the statistics were increased by 11 without degradation of energy resolution from a single counter, and with a degradation in $Q$ resolution from 0.01 to $0.04 \AA^{-1}$.

The results of this procedure is illustrated in figure 4 which shows the energy resolution of incoherent scattering obtained from vanadium foil placed in the same sample holder and in the same geometry as the gallium specimen. The closed circles are the full analysis from eleven counters and the open circles the central counter only. Figure $4 a$ and $4 b$ respectively show the resolution with and without the $12 \mathrm{~mm}$ aperture behind the specimen. In both cases the energy resolution function is found to be very close to a Gaussian profile with a full width at half height of 0.047 and $0.070 \mathrm{THz}$ respectively. The resolution of the spectrometer in momentum space was estimated from the profile of the Bragg's peaks from a solidificated gallium sample to be $\pm 0.01 \AA^{-1}$ for a single counter and $0.04 \AA^{-1}$ for the eleven counters array with corrected energies working in the constant $Q$ mode.

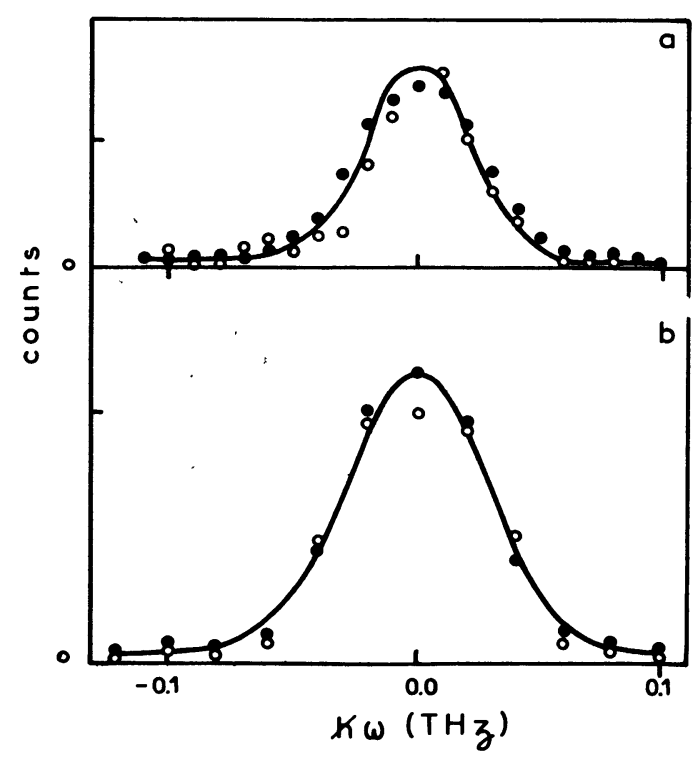

FIG. 4. - Spectrometer energy resolution : the closed circles are the full analysis from eleven counters and open circles the central counter only. Figures $a$ and $b$ respectively show the resolution with and without $12 \mathrm{~mm}$ aperture behind the specimen. The solid lines represent the best fitting Gaussian profiles.
As we shall see the profile of the gallium quasielastic energy distribution at constant $Q$ is observed to be close a Lorentzian function. The observed profile is therefore the Voigt function formed by convoluting this Lorentzian profile with the Gaussian resolution profile. The solid line in figure 5 is a Voigt function fitted to the data by equating the area under the experimental curve to the area under the Voigt function and fitting the Lorentzian width by a least squares procedure.

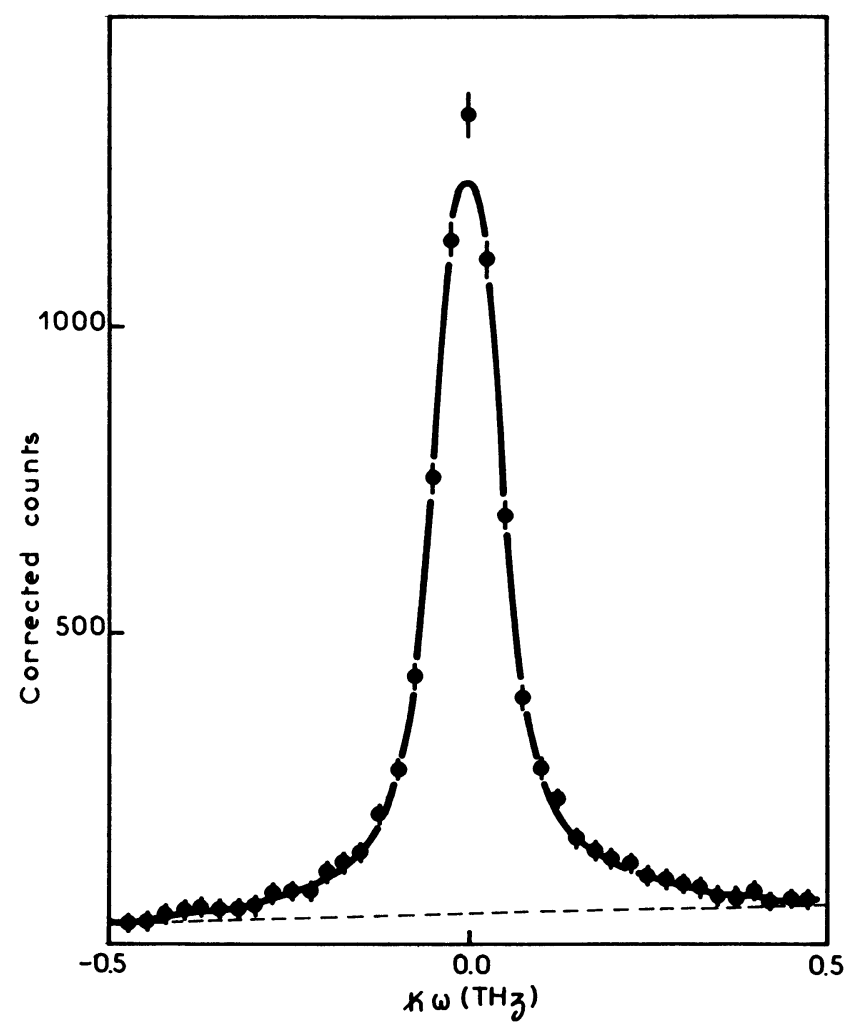

FIG. 5. - Constant $Q$ scan at $Q_{0}=2.50 \AA^{-1}$ in liquid gallium at $213 \mathrm{~K}$. The solid line represents the best fitting Lorentzian convoluted with the experimental resolution function. The fitted background is shown dashed.

3. Presentation of data. - Measurements have been corrected for the container and background scattering and multiplied by appropriate factors to make them proportional to $S(Q, \omega)$ [20]. No correction has been made for multiple scattering and for the $13 \%$ contribution from incoherent scattering in gallium.

We first consider the dependance of the scattering as a function of the wave-vector $Q$. With the analyser set to zero energy transfer, the spectrometer measures the elastic scattering $S(Q, \omega=0)$. In reality because of finite energy resolution, scattered neutron energies within a finite energy window of order $0.05 \mathrm{THz}$ are recorded but, except at the lowest temperatures, the energy width of the scattering is large compared with the resolution width so that the distinction is not significant. The top portion of figure 6 shows this elastic scattering measured by the central counter at two temperatures : above the normal melting point at 
$333 \mathrm{~K}$ and in the supercooling state at $213 \mathrm{~K}$. The same sample was used in each case so the relative intensities are comparable. The curves have the same qualitative form as the total scattering curves measured by X-ray diffraction [11] and shown by full line in the lower part of figure 6. We note however that the elastic scattering presents a more pronounced temperature dependance. The position of the peak in $S(Q, \omega=0)$ at $Q_{0}=2.50 \AA^{-1}$ is identical with that in $S(Q)$ and shows no detectable temperature dependance. The position of the shoulder at $Q_{00}=3.12 \AA^{-1}$ becomes well defined only at lower temperature but is also observed to be temperature independant. The bars on the $S(Q)$ curve represent the integrated areas under our inelastic cross-section measurements : according to eq. (2) this zeroth moment of $S(Q, \omega)$ is identical with $S(Q)$ and the agreement we obtain is a sound check that our inelastic scans within -0.5 and $0.5 \mathrm{THz}$ include the major part of the inelastic scattering.

We next consider these inelastic scans at $213 \mathrm{~K}$ in more detail. This temperature was particularly significant in our experiments since it represents the lowest
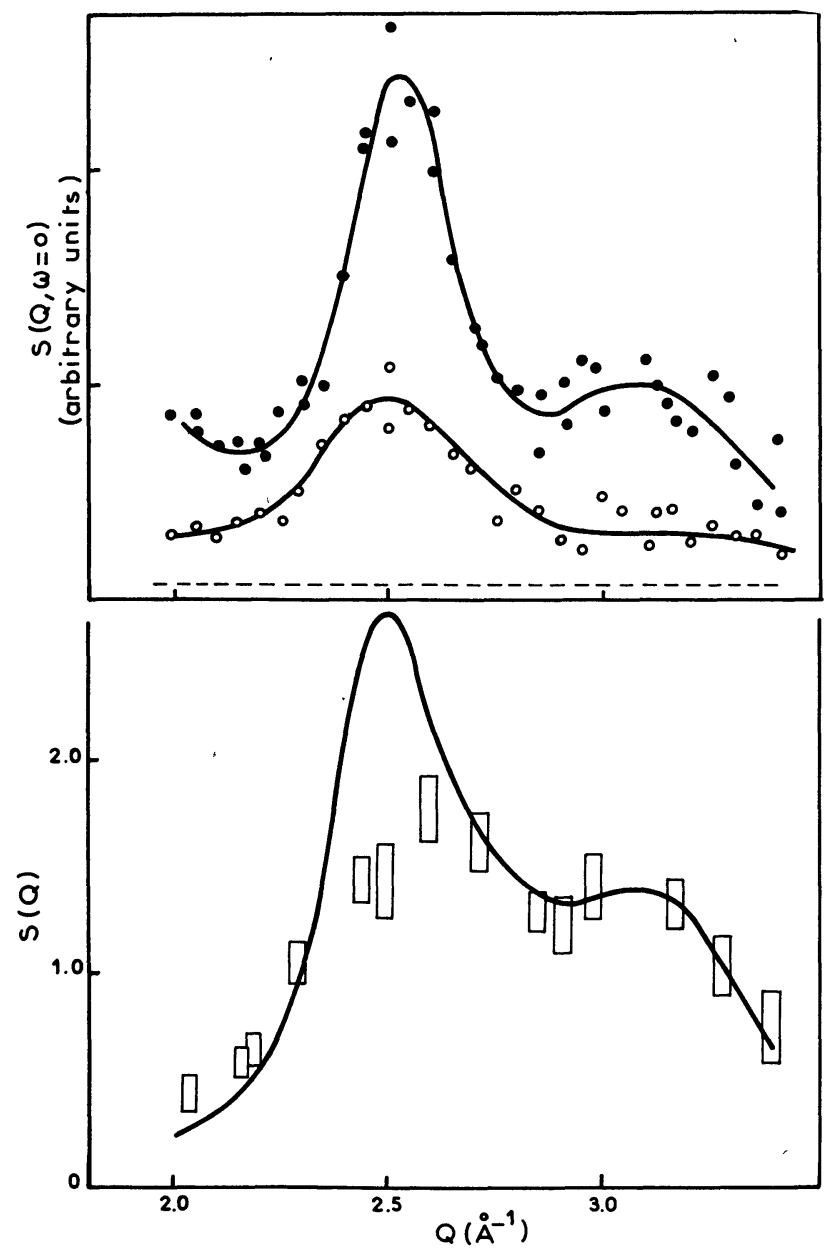

FIG. 6. - Upper part : elastic scattering $S(Q, \omega=0)$ measured by central counter as a function of wave-vector $Q$ at $333 \mathrm{~K}$ (open circles) and at $213 \mathrm{~K}$ (closed circles). Lower part : total scattering $S(Q)$ measured by X-ray diffraction (ref. [10]) at $213 \mathrm{~K}$. The bars represent the integrated area under our quasi-elastic crosssection measurements. temperature at which the sample was completely free from solid droplets. Figure 7 shows the full widths at half height of the frequency distributions measured at constant values of $Q$ between 1.90 and $3.40 \AA^{-1}$. The pronounced dip in the widths near $2.5 \AA^{-1}$ is partly caused by the de Gennes narrowing effect of our eq. (3). The dashed line in figure 7 shows on an arbitrary scale the actual root-mean-square energy width calculated from this equation assuming the free gallium atom mass. Although it has a similar qualitative form in the region of minima, we know that the width of the frequency distribution peak may not be equal to width for a perfect gas ; it is found that for metals high values of $Q \sim 10 \AA^{-1}$ are required before the gas limit is reached [15]. The subsidiary maximum in $S(Q)$ at $Q_{00}=3.12 \AA^{-1}$ is seen only as a small dip in our results while Chen et al. [17] in their study at $301 \mathrm{~K}$ did find a distinct minimum in the widths at $Q_{00}$.

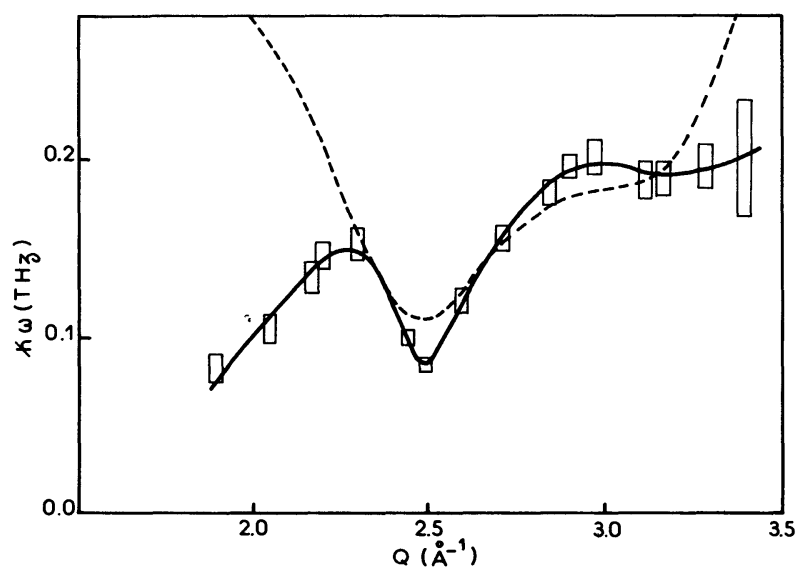

FIG. 7. - Full widths at half height of the frequency distributions from liquid gallium at $213 \mathrm{~K}$ measured at constant value of wavevector $Q$. The dashed line shows the width calculated from eq. (3) in the perfect gas approximation.

Lastly we give in figure 8 the full widths at half height of the frequency distributions at $Q_{0}=2.50 \AA^{-1}$ and $Q_{00}=3.12 \AA^{-1}$ measured as a function of temperature. The data over the temperature range from 163 to $333 \mathrm{~K}$ are clearly consistent with a linear variation of width with temperature. The full line represents a fit to all the data known, giving reduced weights to results from references [16] and [17] which are not consistent with those from reference [18] and the present work. The slope of the line is

$$
\mathrm{d} \omega / \mathrm{d} T=0.0016 \pm 0.0002 \mathrm{THz} . \mathrm{K}^{-1} .
$$

The value for the intercept $T=157 \pm 10 \mathrm{~K}$, although wholly derived from measurements within the liquid phase, is very close to the experimental limit temperature $T_{\mathrm{L}}=150 \mathrm{~K}$ below which it has never been possible to supercool gallium. Width measurements made on the shoulder at $Q_{00}$ give a much larger rate of change with temperature, the slope of the dashed line in figure 8 being equal to about 
$0.0024 \mathrm{THz} . \mathrm{K}^{-1}$; the intercept is however observed to be the same within the much larger statistical error at this $Q$ value.

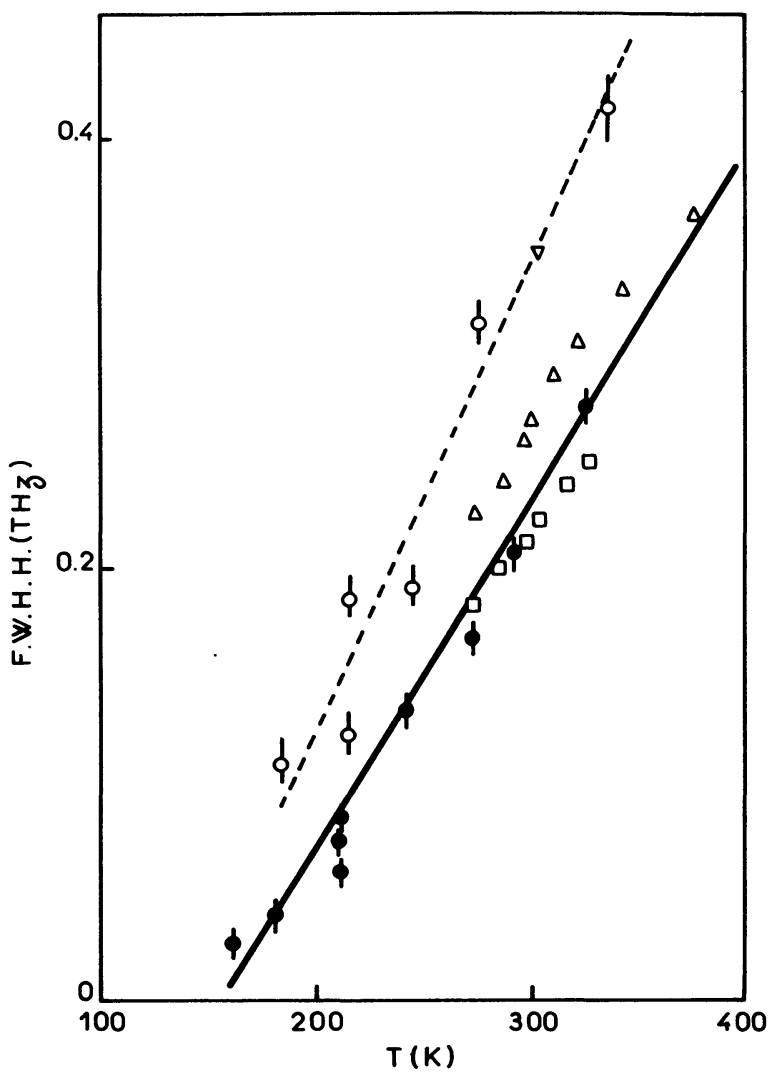

FIG. 8. - Full widths at half height measured as a function of temperature for scans at $Q_{0}=2.50 \AA^{-1}$ (filled circles) and at $Q_{00}=3.12 \AA^{-1}$ (open circles). Previous measurements at $Q_{0}$ are shown by : $\triangle:$ reference [16], $\nabla:$ reference [17], $\square:$ reference [18].

4. Discussion. - Figure 8 shows the quasi-elastic peak widths at $Q_{0}=2.50 \AA^{-1}$ of present work compared with the data obtained by Page et al. [16] in temperature range from 273 to $373 \mathrm{~K}$ and by Hoffner [18] in the temperature range 300 to $1253 \mathrm{~K}$. The widths measured by Chen et al. [17] at $300.4 \mathrm{~K}$ are appreciably greater than ours; this might be caused by the use of time-of-flight data with $Q$ varying notably over the quasi-elastic peak. However taken as a whole the linearity of the dependance of the quasielastic peak width at $Q_{0}$ with temperature is remarkable.
Schneider et al. [6] predicted from mean-field arguments that close to the metastability temperature $T_{\mathrm{L}}$ and wave-vector $Q_{0}$, the quasi-elastic peak would be Lorentzian in form with a full width at half height given by

$$
\begin{aligned}
\text { F.W.H.H. }=Q_{0} \frac{(2 k T)^{1 / 2}}{M} \times & \\
& \times\left[A\left(T-T_{\mathrm{L}}\right)-B\left(Q-Q_{0}\right)^{2}\right] .
\end{aligned}
$$

The Lorentzian lineshape and linear variation near $T_{\mathrm{L}}$ are observed but the linear variation up to the high temperatures observed would not have been expected in view of the approximations of the theory.

From eq. (1) it is readily seen that $S(Q, \omega=0)$ may be written in terms of time-average of the intermediate density correlation function :

$$
S(Q, \omega=0)=\frac{1}{2 \pi} \int \mathrm{d} t\left[\int \exp [i \mathbf{Q} \cdot \mathbf{r}] G(\mathbf{r}, t) \mathrm{d} \mathbf{r}\right]
$$

We mentioned in the introduction the fact that $S(Q)$ is observed to be only very weakly temperature dependant near the metastability limit $T_{\mathrm{L}}$; since $S(Q)$ gives the energy integral of $S(Q, \omega)$, it follows that $S(Q, \omega=0)$ should show a divergence as $T$ approaches $T_{\mathrm{L}}$. The physical reasons for this are not understood but it seems that, as the metastability limit is approached, groups of atoms moving in phase act together to establish an order in the liquid; this ordered state is unstable with respect to infinitesimal fluctuations and crystallisation occurs. The appearance of the $\beta$-phase reflects the fact that the atomic environment in the liquid state is much more similar to the metastable crystalline arrangement than to that in the $\alpha-\mathrm{Ga}$ phase $[11,21]$.

To our knowledge, no theory, certainly not one based on a linear response, is adequate to treat the structural and dynamical phenomena of supercooled gallium described in this work.

Acknowledgments. - We would like to thank Pr. H. Curien for his deep interest in this work. We are indebted to A. Bizid and to the staff of I.L.L. specially R. Scherm, for their help and technical assistance.

\section{References}

[1] Bosio, L. and Windsor, C. G., Phys. Rev. Lett. 35 (1975) $165 \overline{2}$.

[2] Badiali, J. P. and Regnaut, C., Phys. Stat. Sol. (b) 63 (1974) 555.

[3] Bosio, L., Defrain, A. and Epelboin, I., J. Physique 27 (1966) 61.

[4] Nelkin, M. and Raganathan, S., Phys. Rev. 164 (1967) 222.

[5] Kobayashi, K. K., J. Phys. Soc. Japan 27 (1967) 1116.

[6] Schneider, T., Brout, R., Thomas, H. and Feder, J., Phys. Rev. Letì. 25 (1970) 1423.
SCHneIder, T., Phys. Rev. A 3 (1971) 2145

[7] Singwi, K. S., Sköld, K. and Tosi, M. P., Phys. Rev. A 1 (1970) 454.

[8] Leberdev, V. I. and Suchkov, A. A., Ann. Phys. 29 (1973) 302.

[9] Mitra, S. K. and Shukla, G. C., Phys. Stat. Sol. (b) 69 (1974) K33.

[10] Boorstnik, B. and Azman, A., Phys. Stat. Sol. (b) 69 (1975) K19. 
[11] Bizid, A., Bosio, L., Curien, H., Defrain, A. and Dupont, M., Phys. Stat. Sol. (a) 23 (1974) 135.

[12] Carlson, D. G., Feder, J. and Segmuller, A., Phys. Rev. A 9 (1974) 400.

[13] BuCKel, W. and HiLsCh, R., Z. Phys. 138 (1954) 109.

BuCKel, W. and Gey, W., Z. Phys. 176 (1963) 336.

[14] Bererhi, A., Bosio, L., Cortès, R. and Defrain, A., J. Physique Colloq. 36 (1975) C2-79.

[15] Egelstaff, P. A., An introduction to the Liquid State (Acad. Press London) 1967.

[16] Page, D. I., Saunderson, D. H. and Windsor, C. G., J. Phys. C Solid State 6 (1972) 212.
[17] Chen, S. H., Lefevre, Y. and YIP, S., Properties of Liquid Metals (Taylor \& Francis London) 1973, p. 119.

[18] LÖFFLER, U., Dissertation K. F. Karlsruhe.

GläSER, W., HAGen, S., LöfFleR, U., STuCK, J. B. and SCHOMMERS, W., Properties of Liquid Metals (Taylor \& Francis London) 1973, p. 111.

[19] I.L.L. Neutron Beam Facilities Handbook Grenoble, France (1975).

[20] Copley, J. R. D. and Lovesey, S. W., Rep. Prog. Phys. 38 (1975) 461 .

[21] Suzuki, K., Misawa, M. and Fukushima, Y., Trans. Japan Inst. Met. 16 (1975) 297. 